major changes were implemented to improve practice and ensure compliance. Audits play a significant role in optimising the quality of provided patient care. Re-audit is key to assessing and monitoring the effectiveness of the applied measures aiming to continue delivering the best of care to our community.

\section{Quality Improvement and Patient Safety}

\section{THE EFFECTS OF PRE-ADMISSION ISOLATION ON THE EDUCATION AND WELLBEING OF ELECTIVE SURGICAL PATIENTS AND THEIR FAMILIES DURING THE SARS- COV-2 PANDEMIC}

Clare Andrews, Jane Hoddes. Royal National Orthopaedic Hospital

\subsection{6/archdischild-2021-rcpch.586}

Background The SARS-CoV-2 pandemic has significantly impacted all areas of healthcare. Elective surgery ceased during the first lockdown but restarted once SARS-CoV-2 cases decreased, with a strict regime of 14 days pre-admission and post-admission isolation and PCR testing as recommended by Public Health England. In July 2020, RCPCH produced evidence-based recommendations advising that preoperative isolation (PI) was unnecessary in paediatric patients, although this was met with reluctance by many trusts. Our trust revised their guideline with a decrease in PI to 3 days for all but clinically extremely vulnerable (CEV) patients, (based on guidelines covering all age groups, not incorporating $\mathrm{RCPCH}$ criteria), and those requiring elective HDU admission.

We were concerned that children and young people (CYP) were adversely affected, with unnecessary enforced time away from school, impacting on both education and mental health.

Objectives To assess compliance with the trust PI policy that patients and their families were asked to adhere to, and the subsequent impact on education and school attendance and any wider family concerns. We also looked at how this would have differed if $\mathrm{RCPCH}$ vulnerability criteria were used.

Methods Direct questioning of all admitted paediatric patients and their families using a semi structured questionnaire with a mixture of closed and open-ended questions over a 3-week period.

Results A total of 72 patients were admitted during the study period, 47\% (34/72) were asked to preoperatively isolate for 14 days. Of these 11 were classified as ECV using trust guidance, only 3 were ECV by RCPCH criteria. 10 did not fulfil any local criteria. The remainder required elective HDU admission. None of the patients asked to isolate for 3 days fulfilled criteria for longer PI. No patients tested positive for SARS-CoV-2 on PCR during the study period.

- 53 CYP (34 patients and 19 siblings) each missed 10 days off school, totalling 530 lost schooldays.

- 59 CYP in the 3-day PI group missed between 1 and 3 days each (allowing for isolation periods to span weekends). 59177 lost schooldays.
We also saw a significant impact on parents - many reporting financial losses or the use of annual leave or unpaid leave to conform with isolation guidance.

Conclusions Our study showed a discrepancy between clinical practice and trust guidelines, with 14\% of admissions asked to isolate unnecessarily for 14 days.

Our trust guideline did not differentiate between paediatric and adult patients. Following the study, guidance was revised with a separate paediatric pathway using RCPCH CEV criteria to decide between 3 and 14 days PI. HDU admission was removed from high-risk paediatric criteria. Siblings were now exempt from isolation and could attend school.

If our revised trust criteria had been applied to the studied cohort between 428-532 additional schooldays could have been attended (allowing for isolation periods to span weekends).

The impact of SARS-CoV2 pandemic including school closure is well documented to have adversely affected the mental health of CYP. As paediatricians it is important to advocate for our patients and their families ensuring CYP centred care within NHS trusts.

\section{Paediatric Clinical Leaders: Service Planning, Provision and Best Practice}

\section{COACHING FOR TRANSITION: SUCCESS OF A VIRTUAL GROUP COACHING COURSE FOR PAEDIATRIC TRAINEES}

${ }^{1}$ Sarah Davies, ${ }^{2}$ Saranya Ravindran, ${ }^{3}$ Sheena Bailey, ${ }^{4}$ Hina Pattani, ${ }^{5}$ Angela Skidmore, ${ }^{6}$ Trisha Radia. ${ }^{1}$ Chelsea and Westminster Hospital NHS Trust; ${ }^{2}$ Epsom and St Helier NHS Trust; ${ }^{3}$ Sheena Bailey Partnerships; ${ }^{4}$ Guys and St Thomas' Hospital NHS Trust; ${ }^{5}$ Southampton University Hospitals NHS Trust; ${ }^{6}$ Kings College Hospital NHS Foundation Trust

\subsection{6/archdischild-2021-rcpch.587}

Background Coaching is already established as a powerful tool for enhancing executive performance in the corporate sector. There is a growing interest in the role of coaching for the medical workforce.

Coaching aims to facilitate behaviour change, through encouraging self-reflection, challenging automatic thoughts and guiding the coachee to identify the actions required to achieve a desired result.

Few studies report on the effectiveness of coaching as an intervention, however a recent review has shown weak to moderate evidence that coaching is effective for protecting doctor well-being and resilience.

Having previously run a well-received group coaching course for trainee paediatricians returning to work, we were keen to broaden the scope of our course to welcome all trainees approaching transition points in their career and to evaluate their experiences of participation.

Objectives This project aims to evaluate the benefits of a virtual group coaching course for trainee paediatricians facing major career transitions.

Methods Trainee paediatricians were invited to attend virtual group coaching events running in early 2021. The courses were advertised via the regional school website and its 
Whatsapp group. $75 \%$ of places were open to 'self-referring' trainees, whilst $25 \%$ of places were reserved for trainees 'referred' by local training programme directors.

The coaching courses were delivered virtually using zoom software and trainees received a welcome 'package' of precourse information beforehand.

The three-hour sessions were designed and delivered by three professional coaches with experience of working in the healthcare sector.

Each session comprised some short periods of didactic teaching, interspersed with interactive activities performed as a group or in pairs using break out rooms.

Trainees were introduced to the following coaching concepts:

- Listening

- Guided reflection

- Interpersonal dynamics within the workplace

- Contracting

- Peer and self-coaching

Following completion of the course, trainees were requested to complete an online questionnaire to gain an insight into their experience of the course and its most valued elements. Longer term follow-up on the impact of this course for trainees will be sought in the future.

Results 9/14 trainees attending our initial workshop completed our feedback questionnaire. Their baseline data is shown in table 1.

Qualitative feedback has shown several themes emerge in relation to skills learned and projected applications for these.

Trainees particularly valued skills gained in listening, guided reflection and problem solving. They felt these would be useful to them in providing an alternative approach to difficult problems, to supporting junior colleagues and peers and in career related decision making.

\begin{tabular}{lll} 
Abstract 1360 Table 1 & Baseline data & \\
\hline & & $\%$ agree \\
\hline Reason for attending & Transition to SpR & 22 \\
& Transition to Consultant & 33 \\
& Returning after time out & 22 \\
& Other & 22 \\
Very likely to use skills learned & & 66 \\
Found workshop overall useful & & 100 \\
\hline
\end{tabular}

Conclusions Virtually delivered group coaching is valued by paediatric trainees approaching career transitions.

Trainees attending this course were introduced to skills and concepts in listening, reflection and reframing of difficult problems that they anticipate taking forward through their careers, to help negotiate future challenges.

Further feedback will be sought in the coming months pertaining to the real-life impact of trainee participation in this workshop.

\section{British Paediatric Allergy Immunity and Infection Group}

\section{INVESTIGATION OF A LOCAL INCREASE IN RATES OF INVASIVE GROUP B STREPTOCOCCAL INFECTION IN INFANTS; WAS COVID-19 TO BLAME?}

${ }^{1}$ Catherine Baldwin, ${ }^{2}$ Simran Dhariwal, ${ }^{2}$ Lyn Ventilacion, ${ }^{2}$ Rabia Zill-E-Huma, ${ }^{2}$ Eleni Mavrogiorgou, ${ }^{2}$ Sarah Prentice. ' Lister Hospital; ${ }^{2}$ East and North Hertforshire NHS Trust

\subsection{6/archdischild-2021-rcpch.588}

Background Group B streptococcus (GBS) is the most common cause of sepsis and meningitis in infants $<90$ days old in the UK. In 2020 increased rates of invasive GBS (iGBS) were noted in infants at a district general hospital in the East of England. A multi-disciplinary review was conducted to try to identify reasons for this increase, and to determine whether systems changes due to the COVID-19 pandemic may have played a role.

Objectives Investigation of a local increase in invasive GBS cases in infants.

Methods A retrospective case note review of all cases of iGBS occurring between 2015 and 2020 was conducted. Cases occurring in 2020 were compared to cases in 2015-2019 and to a matched-control population for presence of known risk factors and demographics, Whole genome sequencing was conducted on bacterial isolates from cases in 2020 in order to identify whether the increased incidence represented an outbreak.

Results In 202014 cases of iGBS occurred in infants $<90$ days of age (Incidence rate (IR) 2.55/1000); 5 Early Onset GBS (EOGBS, 0-6d, IR 0.91/1000)) and 9 Late Onset GBS (LOGBS, 7-89d, (IR 1.64/1000)) at our hospital. These rates were 1.2 -fold and 4.5 -fold higher, respectively, than the local rates for 2019, and 1.7-fold and 5.5-fold higher than the 2019 national rates.

All babies with EOGBS in 2020 had identifiable risk factors; $4 / 5$ had maternal pyrexia $>38^{\circ} \mathrm{C}, 1 / 5$ had suspected chorioamnionitis and $3 / 5$ had prolonged rupture of membranes $>18$ hours. All received appropriate intra-partum antibiotics. GBS colonisation was unknown prior to delivery in any mother, with $60 \%$ of mothers in 2020 subsequently testing positive compared to $27 \%$ in 2015 2019. There were no significant differences in the demographics of infants with EOGBS in 2020 compared to 2015-2019. All cases of EOGBS in 2020 were identified rapidly following delivery, and treated according to NICE guidelines.

Cases of LOGBS in 2020 tended to occur more in low risk, term deliveries compared to previous years. In 2020 $43 \%$ of infants with LOGBS had no identifiable risk factors for LOGBS at delivery, compared to $23 \%$ in the early timeperiod. Infants with LOGBS in 2020 were demographically comparable to those in 2015-2019.

No clear evidence of an impact of the COVID-19 pandemic was found. Mothers of infants with LOGBS disease had slightly fewer antenatal microbiological samples, where GBS 